\title{
BioGlass: Physiological Parameter Estimation Using a Head-mounted Wearable Device
}

\author{
Javier Hernandez ${ }^{1}$ \\ ${ }^{1}$ Media Lab \\ Massachusetts Institute of Technology \\ Cambridge, MA, USA \\ \{javierhr, picard\}@media.mit.edu
}

\author{
James M. Rehg ${ }^{2}$ \\ ${ }^{2}$ School of Interactive Computing \\ Georgia Institute of Technology \\ Atlanta, GA, USA \\ \{yli4400,rehg\}@gatech.edu
}

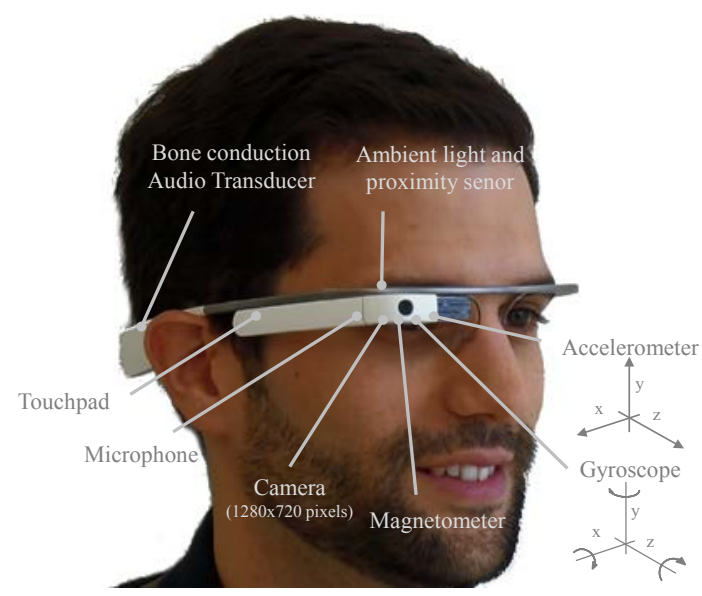

\begin{abstract}
This work explores the feasibility of using sensors embedded in Google Glass, a head-mounted wearable device, to measure physiological signals of the wearer. In particular, we develop new methods to use Glass's accelerometer, gyroscope, and camera to extract pulse and respiratory rates of 12 participants during a controlled experiment. We show it is possible to achieve a mean absolute error of 0.83 beats per minute (STD: 2.02) for heart rate and 1.18 breaths per minute (STD: 2.04) for respiration rate when considering different combinations of sensors. These results included testing across sitting, supine, and standing still postures before and after physical exercise.
\end{abstract}

Ballistocardiogram (BCG); blood volume pulse (BVP); heart rate; respiration rate; head-mounted wearable device; gyroscope; accelererometer; camera; daily life monitoring.

\section{INTRODUCTION}

Being able to comfortably monitor physiological information during daily life can reduce the costs associated with health care [16]. Physiological measures such as cardiovascular and respiratory activity can be used for early detection and diagnosis of relevant risk factors of cardiovascular disease [2] as well as for helping to monitor chronic conditions and therapeutic interventions.

Traditional approaches to measure parameters such as heart rate require attaching electrodes to the skin, which is cumbersome for daily life monitoring. However, recent advances in technologies have enabled the creation of wearable devices of reduced sized, weight and power consumption. These devices are in close contact with the body and offer a new set of low-cost unobtrusive sensors that can run continuously during daily activities. A recent commercial effort is Google Glass (see Fig. 1). Google Glass is a wireless headmounted device equipped with a touch pad, a see-through display, and most of the sensors available in smartphones. Although the device was not designed for physiological measurement, its unique location on the head of the person provides an opportunity to unobtrusively and continuously monitor physiological information during daily activities. In this work we develop new methods allowing the gyroscope, the accelerometer and the camera embedded in Glass to be used to capture subtle head motions of the wearer that are associated with the mechanical activity of the heart (a.k.a., ballistocardiography) and the respiration of the wearer.

This material is based upon work supported by Google, the National Science Foundation (NSF 1029585 and NSF 1029679), and the MIT Media
Figure. 1. Head-mounted wearable device Google Glass and the locations of some of the main sensors.

\section{PREVIOUS WORK}

Ballistocardiography was popularized by Starr et al [15] who showed that the mechanical ballistic forces of the heart elicit subtle body movements. While the original experiments required a subject to lie down on a suspended supporting structure, continuous technological advances have enabled BCG measurement in less constrained settings. For instance, Kown et al [9] and Dinh [3] attached a smartphone to the chest and used its accelerometer to monitor heart rate. Similarly, Phan et al [13] proposed a different approach to extract heart rate and respiration from chest motion. Other researchers have also successfully gathered BCG information from modified daily life devices such as a weighing scale [8]. In a separate study, He et al [7] developed an ear-worn device that used an accelerometer to reliably extract heart rate of participants under different conditions. In [6], He also showed preliminary results of estimating respiration from accelerometer data for a single sample but no validation was performed. Our work also includes heart rate estimation from accelerometer data and provides a methodological validation of respiration rate estimation. Furthermore, we show improvements with novel use of the gyroscope and camera, and explore a new location of the sensor (above the right eye instead of over the ear). Our work also makes use of a head-worn camera that captures the egocentric view of the person to monitor subtle periodic 

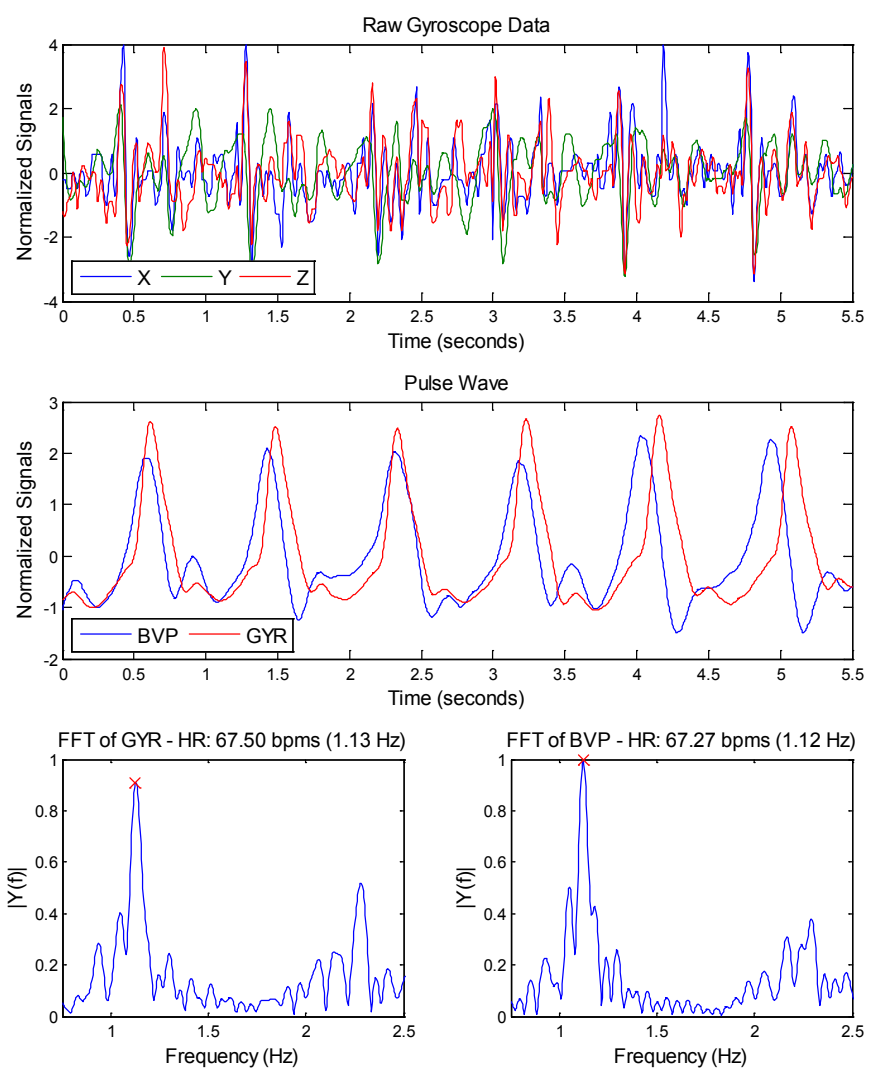

Figure. 2. Example of an estimated pulse wave from gyroscope data (red) and the ground truth blood volume pulse signal (blue). Bottom graphs show the Fourier Spectrum of each signal. (FFT: Fourier Spectrum, GYR: Gyroscope, BVP: Blood Volume Pulse, HR: Heart Rate, bpms: beats per minute)

motions. This is in contrast to the work of Balakrishnan et al [1], which measured the heart rate of a person in front of a static camera by monitoring subtle BCG head motions. To the best of our knowledge, this work is the first to use the egocentric view of a wearer to gather his or her own physiological data.

\section{EXPERIMENTAL PROTOCOL AND APPARATUS}

Twelve participants (6 females) with an average age of 27.3 (STD of 5.3) years old, weight of 144.5 (STD: 30.9) pounds and height of 5.65 (STD: 0.4) feet participated in this study. After obtaining written consent, participants were asked to keep still, breathe spontaneously and look at a static indoor scene situated at a distance of 2.2 meters while remaining in three different positions (standing up, sitting down and lying down) for a minute each. In order to generate a larger dynamic range of physiological readings, participants were then asked to repeat the three positions after pedaling a stationary bike for one minute. Ground truth physiology was measured with an FDA cleared sensor (FlexComp Infiniti by Thought Technologies) that simultaneously recorded Blood Volume Pulse (BVP) from the finger and respiration from a chest belt sensor at a constant sampling rate of $256 \mathrm{~Hz}$. The Institutional Review Board of the Massachusetts Institute of Technology approved the study and participants were compensated with a \$5 Amazon gift card.
We created a custom Android application to simultaneously $\log$ information from the accelerometer, the gyroscope and the camera of an early beta version of Google Glass. Both 3-axis accelerometer and 3-axis gyroscope were retrieved at an average sampling rate of $50 \mathrm{~Hz}$. In order to ensure a constant sampling rate, we performed cubic interpolation at a sampling rate of $256 \mathrm{~Hz}$ (the same as the FlexComp Infiniti sensor). There were some sporadic cases where the sensor did not log any data for long periods of time (e.g., half a second), which introduced critical artifacts when estimating physiological information. To minimize the effect of these artifacts, we applied a hard-thresholding method (2 STD above and below the mean) for each observation window. The video was recorded at a constant frame rate of $30 \mathrm{~Hz}$ at a resolution of $1280 \times 720$ pixels (the default settings of Glass). Each of the pixels yields a vector in RGB color space. Motion was estimated by tracking 2D feature points in the video. First, we detected feature points [14] in each frame and tracked them using a Kanade-Lucas-Tomasi feature tracker [11]. We then fit a homography matrix [5] to the point correspondences using RANSAC [4]. We assume that all tracked points correspond to static 3D points, in which case their offsets are solely explained by the camera motion. Finally, the vertical and horizontal motion (up to a scale) of the camera can then be directly extracted from the matrix.

\section{Physiological PARAmeter Estimation}

A challenge in extracting physiological parameters during daily activity with wearable devices is to develop algorithms that require low-computational power and run in real-time. Therefore, we constrained our new methods to use combinations of efficient signal processing techniques.

Given a specific sensor modality with sensor readings as a time series of vectors (e.g., 3D vector for accelerometer and gyroscope, 2D vector for camera), the estimation of the pulse wave was divided into the following steps:

1) A moving average window of 3 samples was subtracted from each dimension of the vector, allowing the removal of signal shifts and trends.

2) A band-pass Butterworth filter of order 4 with cut-off frequencies of 10 and $13 \mathrm{~Hz}$ was applied to each dimension to isolate BCG changes.

3) In order to aggregate the different components of the signal, i.e. dimensions of the vector, we compute the square root of the summation of the squared components (i.e., L2 norm) at each sample. This aggregation gives the same relevance to each of the dimensions and makes our approach more robust to different body postures.

4) Finally, a band-pass Butterworth filter of order 2 with cutoff frequencies of 0.75 and $2.5 \mathrm{~Hz}$ (corresponding to 45 and 150 beats per minute) was applied, yielding the final pulse wave.

Fig. 2 shows an example of pulse wave estimation from gyroscope data of a person wearing the head-mounted wearable device while lying down. As can be seen, the estimated pulse wave is well aligned with the wave of reference. 
TABLE I. HEART RATE ESTIMATION

\begin{tabular}{|c|c|c|c|c|}
\hline Sensor & ME & STD & RMSE & CC \\
\hline Gyroscope & 0.83 & 2.02 & 2.19 & 0.99 \\
\hline Accelerometer & 2.41 & 6.45 & 6.88 & 0.92 \\
\hline Camera & 7.89 & 13.35 & 15.50 & 0.59 \\
\hline All & 1.21 & 3.45 & 3.66 & 0.98 \\
\hline
\end{tabular}

TABLE II. RESPIRATION RATE ESTIMATION

\begin{tabular}{|c|c|c|c|c|}
\hline Sensor & ME & STD & RMSE & CC \\
\hline Gyroscope & 1.39 & 2.29 & 2.67 & 0.75 \\
\hline Accelerometer & 2.26 & 3.38 & 4.07 & 0.43 \\
\hline Camera & 1.58 & 2.62 & 3.06 & 0.68 \\
\hline All & 1.18 & 2.04 & 2.36 & 0.79 \\
\hline
\end{tabular}

$\mathrm{ME}=$ Mean absolute error, STD $=$ Standard deviation of the absolute error, $\mathrm{RMSE}=$ Root mean squared error, $\mathrm{CC}=$ Pearson's correlation coefficient.

In order to estimate the respiratory wave, we performed the following steps independently for each sensor modality:

1) An averaging filter was applied to each of the components. The window length was set to be the duration of a respiration cycle at a maximum breathing rate ( 45 breaths per minute in our case).

2) A band-pass Butterworth filter of order 4 with cut-off frequencies of 0.13 and $0.75 \mathrm{~Hz}$ (corresponding to 8 and 45 breaths per minute) was applied to each dimension.

3) Since different dimensions of the sensor reading may change in different directions depending on the body position, we used Principal Component Analysis to transform the data into a set of components that maximize the variance. After this transformation, we computed the Fast Fourier Transform of each new component and selected the most periodic signal. The periodicity of the signal was estimated by computing the maximum amplitude observed within the previous frequency range.

For each of the waves we extracted the heart rate and the respiration rate in the frequency domain. Given an estimated wave or ground truth signal, we extracted the frequency response with the Fast Fourier Transform and identified the frequency with the highest amplitude response. The band of frequencies used for the pulse and respiration rates are the same ones considered in the previous section. The estimated heart rate and respiration rate corresponded to the maximum frequency multiplied by 60 (see bottom graph of Fig. 2).

\section{RESULTS AND DISCUSSION}

Each of the 12 participants held three different positions under relaxed and aroused (after biking) conditions for a minute each. Therefore, we collected 72 1-minute segments of data. In order to perform the analysis, we divided the data into intervals of 20 seconds with a $75 \%$ overlap (n: 576 samples).

\section{A. Comparison across Modalities}

To evaluate the utility of each sensor modality, we extracted heart rate and respiration rate from each of the samples and
TABLE III. MEAN ABSOLUTE ERROR OF HEART RATE

\begin{tabular}{|c|c|c|c|}
\hline Sensor & Sitting & Standing & Supine \\
\hline Gyroscope & 1.15 & 0.91 & 0.44 \\
\hline Accelerometer & 3.30 & 1.71 & 2.22 \\
\hline Camera & 4.42 & 10.45 & 8.80 \\
\hline All & 1.49 & 1.16 & 0.98 \\
\hline
\end{tabular}

TABLE IV. MEAN ABSOLUTE ERROR OF RESPIRATION RATE

\begin{tabular}{|c|c|c|c|}
\hline Sensor & Sitting & Standing & Supine \\
\hline Gyroscope & 1.14 & 1.97 & 1.07 \\
\hline Accelerometer & 1.90 & 3.08 & 1.80 \\
\hline Camera & 1.35 & 1.88 & 1.51 \\
\hline All & 0.98 & 1.77 & 0.80 \\
\hline
\end{tabular}

computed several performance metrics. Tables I and II show a summary of the metrics for heart rate and respiration rate, respectively, across all the 576 segments ( 72 minutes) of data computed from the 12 participants. As can be observed, there is a close agreement between the measurements and groundtruth, providing evidence to the feasibility of the proposed methods. When comparing the three sensors individually, the gyroscope yielded the best performance for both heart and respiration rates, achieving a mean absolute error of 0.83 beats per minute (STD: 2.02) and 1.39 breaths per minute (STD: 2.29), respectively. Notably, the accelerometer (upon which prior BCG work is based) was never the best performing modality for heart rate or respiration.

The process of extracting motion measurement from video is more complex and less direct than it is for the other two sensors. For instance, different factors such as the depth of the scene or the amount of feature points that can be tracked in the environment have a direct impact on the performance. Furthermore, the sampling rate of the camera was significantly lower than the other two sensors. Therefore, high frequency changes such as subtle head movements due to heart activity may not be as accurately captured as the low frequency movements associated with respiration.

\section{B. Postural Changes}

Body posture mediates the intensity and quality of BCG movements. In this study, participants were measured from three body postures: sitting, standing, and supine. Tables III and IV show the mean absolute error for each of the different positions and sensors. When estimating heart rate, the most challenging position was sitting down, which is in accordance with the results described in [6]. The results obtained with the gyroscope in this study outperform their results for the sitting (ME: 1.27) and supine (ME: 0.84) conditions but not for the standing (ME: 0.72) position. However, the range of heart rates they observed in their study was considerably smaller (55 to 95 beats per min.) in comparison to our experiment (56 to 133 beats per min.). The different results for the camera sensor may be due to a combination of several factors such as the influence of body posture, the accuracy of motion estimation 
as well as the relative pose of the camera with respect to the wearer's head. When estimating respiration rate, the most challenging position was standing up for all the modalities. Apparently, respiratory movements have less influence on head motion while standing. Overall, even the most challenging posture positions yielded low error for the bestperforming modality.

\section{Combination of Modalities}

Differences in performance across modalities are partly due to the different types of information being captured by each of the sensors. For instance, while the accelerometer data captures linear accelerations, the gyroscope captures rotations of the device. Furthermore, while some of the sensors may be affected by sampling rate artifacts (e.g., accelerometer and gyroscope), other sensors may provide more constant sampling rates but less accurate information (e.g., camera). Therefore, a combination of different modalities may help to provide more reliable estimates at the cost of computational complexity. To start exploring this idea, we extracted the heart/respiration rates of each modality separately and computed the median as the final estimate. The bottom rows of Tables I and II show the results using this late fusion technique. While the heart rate estimation using the gyroscope was still better than the one obtained combining all the modalities, the respiration rate estimation with all three modalities yielded better results than with any of the other modalities alone (reducing the ME to 1.18 breaths per minute).

\section{CONCLUSIONS}

In this work we have explored the possibility of using different sensor modalities of a head-mounted wearable device to extract physiological parameters of the wearer. We have proposed two new real-time algorithms and demonstrated their effectiveness in a controlled laboratory setting for estimating heart and respiration rates. Furthermore, we have provided a quantitative comparison across modalities and body postures.

Among the three modalities, the use of a gyroscope outperformed the other sensors, including the accelerometer upon which prior BCG measurement is based. We believe this improvement is partly due to the above-eye location of the sensor and its capability to capture amplified rotational movements of BCG. The ego-centric head-mounted camera has shown to be a novel and promising method to harvest physiological information of the wearer. While the camera requires significantly more energy than the other two sensors, it offers the opportunity of capturing insightful visual context that helps interpret the physiological changes. Furthermore, ego-centric videos are usually more available on the web than accelerometer and gyroscope readings. We expect different modalities to complement each other during real-life monitoring as different sensors may capture different types of movement. Future efforts will focus on the development of sophisticated methods that can handle large motions associated with daily activities and the collection of more naturalistic samples, which is fundamental to apply the proposed methods in real world settings.

In summary, this work has shown a new capability to provide accurate real-time heart-rate and respiration measures from a gyroscope worn above the eye, and using the combination of sensors available in today's head-mounted wearable Google Glass. While the current work has focused on three different postures when the wearer is holding relatively still, future work can now expand the capabilities to active daily wear. With the continuous technological improvements and commercial reach of new devices, we expect our results will help facilitate non-intrusive access of meaningful physiological information during daily activity.

\section{REFERENCES}

[1] G. Balakrishnan, F. Durand, and J. Guttag, "Detecting Pulse from Head Motions in Video." IEEE Conference on Computer Vision and Pattern Recognition (CVPR), 2013.

[2] S. Cook, M. Togni, M. C. Schaub, P. Wenaweser, and O. M. Hess, "High heart rate: a cardiovascular risk factor?" Eur. Heart J., 27 (20), pp. 2387-93, 2006.

[3] A. Dinh, "Heart Activity Monitoring on Smartphone," International Conference on Biomedical Engineering and Technology, 11, pp. 45-49, 2011.

[4] M. A. Fischler and R. C. Bolles. "Random sample consensus: a paradigm for model fitting with applications to image analysis and automated cartography," Communications of the ACM, 24 (6), pp. 38195, 1981.

[5] R. Hartley and A. Zisserman. "Multiple view geometry in computer vision." Cambridge university press, 2004.

[6] D. D. He, "A wearable heart monitor at the ear using ballistocardiogram (BCG) and electrocardiogram (ECG) with a nanowatt ECG heartbeat detection circuit," Ph.D. dissertation, Dept. Elect. Eng. and Comp. Scie., Massachusetts Institute of Technology, Cambridge, MA, 2013.

[7] D. D. He, E. S. Winokur, and C. G. Sodini, "An ear-worn continuous ballistocardiogram (BCG) sensor for cardiovascular monitoring," Conf. Proc. IEEE Eng. Med. Biol. Soc., vol. 2012, pp. 5030-3, Jan. 2012.

[8] O. T. Inan, M. Etermadi, R. M. Wiard, L. Giovangrandi, G. T. Kovacs, "Robust ballistocardiogram acquisition for home monitoring," Physiological measurement, 30 (2), pp. 169-85, 2009.

[9] S. Kwon, J. Lee, G. S. Chung, and K. S. Park, "Validation of heart rate extraction through an iPhone accelerometer.," Conf. Proc. IEEE Eng. Med. Biol. Soc., vol. 2011, pp. 5260-3, Jan. 2011.

[10] C. Link, D. Da He, E. S. Winokur, T. Heldt, and C. G. Sodini, "The Ear as a Location for Wearable Vital Signs Monitoring," In Conf Proc IEEE Eng Med Biol Soc., 2010.

[11] B. D. Lucas and T. Kanade. "An Iterative Image Registration Technique with an Application to Stereo Vision," International Joint Conference on Artificial Intelligence, pp. 674-679, 1981.

[12] Z. B. Moses, L. J. Luecken, and J. C. Eason, "Measuring task- related changes in heart rate variability," in Engineering in Medicine and Biology Society, pp. 644-647, 2007.

[13] D. H. Phan, S. Bonnet, R. Guillemaud, E. Castelli, and N. Y. P. Thi, "Estimation of respiratory waveform and heart rate using an accelerometer," Engineering in Medicine and Biology Society (EMBS), 30th Annual International Conference of the IEEE, pp. 4916-9, 2008.

[14] J. Shi and C. Tomasi. "Good features to track," IEEE Conference on Computer Vision and Pattern Recognition (CVPR), pp. 593-600, 1994.

[15] I. Starr, A. J. Rawson, H. A. Schroeder, and N. R. Joseph, "Studies on the estimation of cardiac output in man, and of abnormalities in cardiac function, from the hearts recoil and the bloods impacts; the ballistocardiogram," The American J. of Physiology, 127 (1), 1939.

[16] G. Troster, "The agenda of wearable healthcare," IMIA Yearbook of Med. Info. 2005: Ubiquitous Health Care Sys, pp. 125-138, 2004. 